\title{
Performance of a resistive plate chamber equipped with a new prototype of amplified front-end electronics
}

\author{
Massimiliano Marchisone ${ }^{a, b}$ on behalf of the ALICE Collaboration \\ ${ }^{a}$ iThemba LABS, National Research Foundation, PO Box 722, Somerset West, South Africa \\ ${ }^{b}$ University of the Witwatersrand, 1 Jan Smuts Avenue, Braamfontein, Johannesburg, South Africa \\ E-mail:mmarchis@cern.ch
}

ABSTRACT: ALICE is the LHC experiment dedicated to the study of heavy-ion collisions. At forward rapidity a muon spectrometer detects muons from low mass mesons, quarkonia, open heavy-flavor hadrons as well as weak bosons.

A muon selection based on transverse momentum is made by a trigger system composed of 72 resistive plate chambers (RPCs). For the LHC Run 1 and the ongoing Run 2 the RPCs have been equipped with a non-amplified FEE called ADULT. However, in view of an increase in luminosity expected for Run 3 (2021-2023) the possibility to use an amplified FEE has been explored in order to improve the counting rate limitation and to prevent the aging of the detector, by reducing the charge per hit. A prototype of this new electronics (FEERIC) has been developed and tested first with cosmic rays before equipping one RPC in the ALICE cavern with it.

In this paper the most important performance indicators - efficiency, dark current, dark rate, cluster size and total charge - of an RPC equipped with this new FEE will be reviewed and compared to the others read out with ADULT, in pp collisions at $\sqrt{s}=5$ and $13 \mathrm{TeV}$ and in $\mathrm{Pb}-\mathrm{Pb}$ collisions at $\sqrt{s_{\mathrm{NN}}}=5 \mathrm{TeV}$.

KEywords: Resistive-plate chamber, Trigger detector, Muon spectrometer

ARXIV EPRINT: 1605.08537 


\section{Contents}

1 Introduction 1

2 First results with cosmic rays 2

3 FEERIC in ALICE 2

3.1 Working point determination 3

3.2 Efficiency stability 3

3.3 Dark current and dark rate time dependence 4

3.4 Evaluation of the charge reduction with FEERIC 5

4 Conclusions and perspectives $\quad 5$

\section{Introduction}

ALICE [1] is a multitasking experiment at the CERN-LHC, mainly designed to study the hot and dense matter produced in heavy-ion collisions. At forward rapidity $(2.5<y<4)$ muons are detected and tracked with a muon spectrometer [2]. It consists of a set of absorbers, a dipole magnet, a muon tracking system and a muon trigger detector [3].

The muon trigger system consists of 72 Resistive Plate Chambers (RPCs) [4] arranged in two stations at a distance of 16 and $17 \mathrm{~m}$ from the interaction point. Each station is made of two detection planes with 18 RPCs each, perpendicular to the beam line. The chambers are $2 \mathrm{~mm}$ single gap RPC, with low resistivity $\left(\sim 10^{9} \Omega \cdot \mathrm{cm}\right)[5]$ bakelite electrodes and are operated in the so-called "maxi-avalanche" mode [6], with a gas mixture consisting of $89.7 \%$ $\mathrm{C}_{2} \mathrm{H}_{2} \mathrm{~F}_{4}, 10 \% \mathrm{C}_{4} \mathrm{H}_{10}$ and $0.3 \% \mathrm{SF}_{6}$. The gas relative humidity is kept at $37 \%$ to prevent alterations in the bakelite resistivity [7]. The operating high voltages are optimized for each $\mathrm{RPC}$ and range from 10.0 to $10.4 \mathrm{kV}$ [8]. The signal is picked up inductively on both sides of the detector by means of orthogonal copper strips $(1,2$ and $4 \mathrm{~cm}$ ), in the horizontal (bending plane) and vertical (non-bending plane) directions. With the present FEE (ADULT ASIC [9]) the signal is discriminated without pre-amplification, with thresholds set at $7 \mathrm{mV}$ and the mean charge per hit produced in the gas gap is of the order of $100 \mathrm{pC} \mathrm{[10].}$

In those conditions, the instantaneous counting rate limit is $50 \mathrm{hits} / \mathrm{s} / \mathrm{cm}^{2}$, including some safety margins. Furthermore, the safe operation of the detectors cannot be guaranteed for a cumulative charge larger than $50 \mathrm{mC} / \mathrm{cm}^{2}$ (i.e. $500 \mathrm{Mhits} / \mathrm{cm}^{2}$ in maxi-avalanche) [6]. These two limitations are not compatible with the expected conditions after the second LHC long shutdown (2019-2020). Indeed, peaks of counting rates up to $125 \mathrm{hits} / \mathrm{s} / \mathrm{cm}^{2}$ and a cumulative charge of $100 \mathrm{mC} / \mathrm{cm}^{2}$ should be reached by the most exposed RPCs in central $\mathrm{Pb}-\mathrm{Pb}$ collisions. 
A possible solution for overcoming these two problems is to operate the RPCs at lower gain, like in ATLAS $[11,12]$ and in CMS $[13,14]$. This requires an upgrade of electronics with amplification to reduce the charge per hit. The proposed solution is to replace the present front-end boards with new ones based on the FEERIC ASIC (Front-End Electronics Rapid Integrated Circuit) developed at Clermont-Ferrand (France) [15]. Unlike ADULT, FEERIC performs amplification of the analog signals from the RPC before discrimination. This should allow one to reduce by a factor 3-5 the charge produced in the gas, hence limiting the aging effects.

\section{First results with cosmic rays}

Preliminary measurements have been performed with FEERIC board prototypes for strips of $2 \mathrm{~cm}$ at the beginning of 2015, using the test bench available in Torino (Italy) [16]. The $\mathrm{RPC}$ under test has been filled with the same gas mixture and relative humidity adopted in the muon trigger.

Efficiency curves with FEERIC for various discrimination thresholds are compared in the left figure 1, to the one obtained with ADULT with $7 \mathrm{mV}$ threshold. The rise to the efficiency plateau is found to be quite sharp and a clear shift towards lower high voltage working points can be observed with FEERIC. The HV shift depends strongly on the FEERIC discrimination threshold, which is limited by the environmental noise conditions, however the HV shift is not less than 600-700 V.
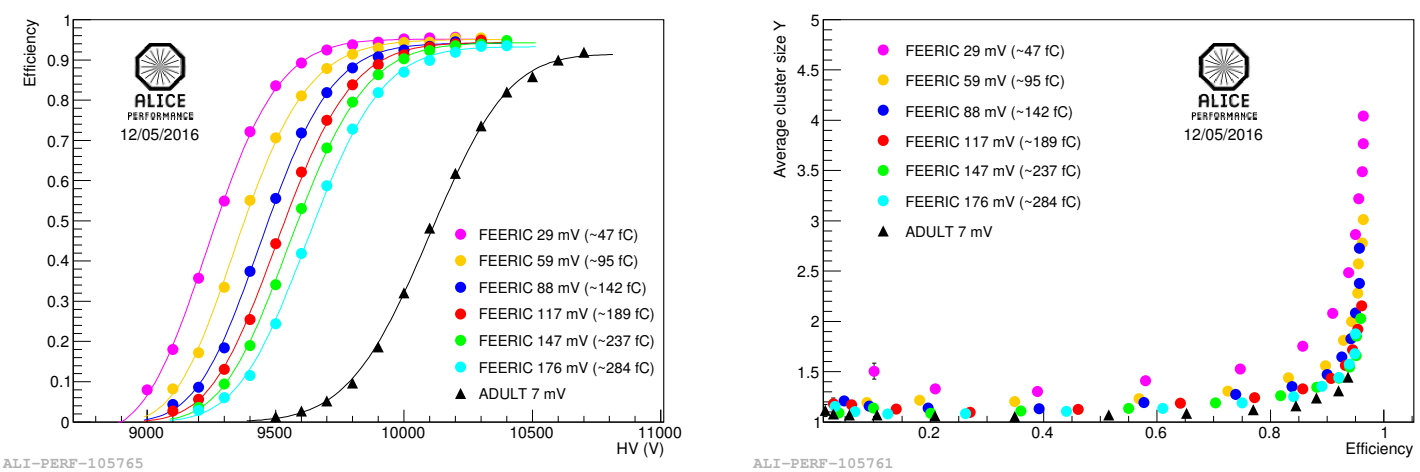

Figure 1. Efficiency curve (left) and cluster size measurements for strips of $2 \mathrm{~cm}$ (right) from cosmic data taking. Colors refer to different discrimination thresholds.

The cluster size as a function of the RPC efficiency is shown in figure 1 (right). In general the average value measured at the working point with FEERIC is slightly higher than what is measured with ADULT. This can be explained by considering the different signal amplitude distributions in the two working modes.

\section{FEERIC in ALICE}

From the results described above, it is however very difficult to anticipate the achievable charge gain in the experimental working conditions. This is why it was foreseen to equip 
one of the 72 RPCs in the ALICE cavern with FEERIC electronics with final ASIC, in order to evaluate the performance during LHC Run 2. In February 2015, 39 cards were installed on one RPC with strips of 2 and $4 \mathrm{~cm}$, placed in the fourth plane of the muon trigger. The performance in terms of efficiency, cluster size, current, single counting rate, stability and robustness are now discussed exploiting the 2015 data taking in pp collisions at $\sqrt{s}=5$ and $13 \mathrm{TeV}$ and in $\mathrm{Pb}-\mathrm{Pb}$ collisions at $\sqrt{s_{\mathrm{NN}}}=5 \mathrm{TeV}$.

\subsection{Working point determination}

The working point of the RPC equipped with FEERIC cards has been determined by means of a HV scan performed in June 2015 during low luminosity pp runs at $13 \mathrm{TeV}$, for three thresholds. The results are shown in figure 2 (left). Full efficiency is reached for all threshold values and the results are very similar for positive (bending plane) and negative (non-bending plane) polarities.
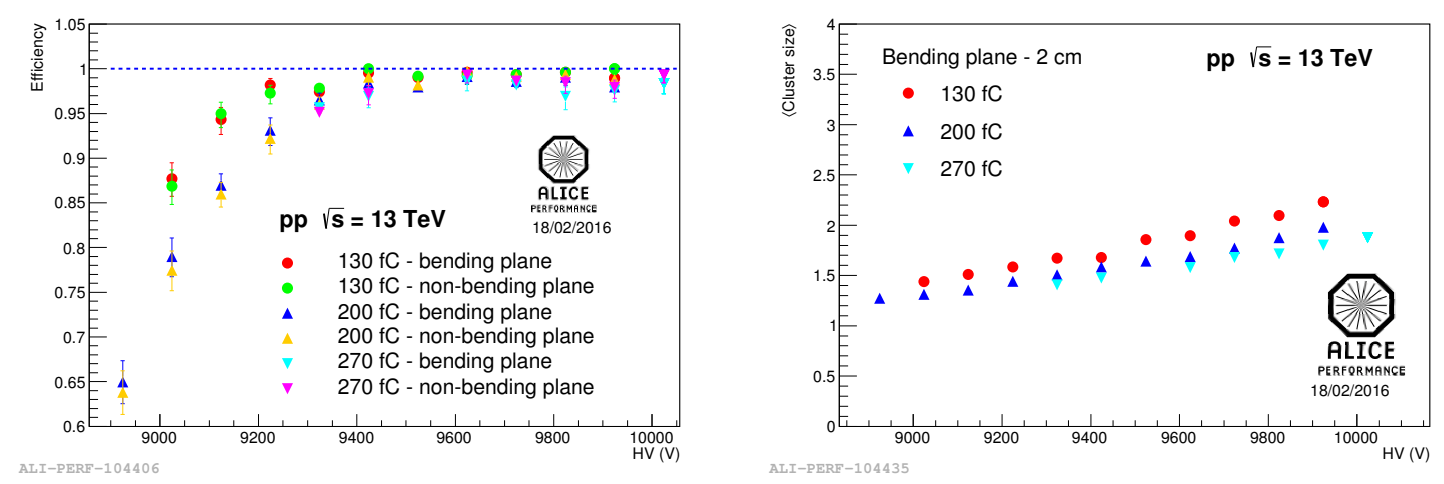

Figure 2. Efficiency plateau (left) and cluster size measurements for strips of $2 \mathrm{~cm}$ (right) in pp collisions at $13 \mathrm{TeV}$. Colors refer to different discrimination thresholds.

The HV dependence of the cluster size for strips of $2 \mathrm{~cm}$ is also shown in figure 2 (right). Measurements are larger for the lower threshold values and increase with HV. As already noticed during the tests with cosmic rays, in maxi-avalanche mode (with ADULT cards with a threshold of $7 \mathrm{mV}$ ) the cluster size is lower, $\sim 1.4$ for strips of $2 \mathrm{~cm}$ width [10].

By considering these results and the fact that no noise is observed in ALICE cavern for a threshold voltage above $33 \mathrm{mV}(\sim 60 \mathrm{fC})$ for all FEERIC card formats and polarities, the working point has been set at $9375 \mathrm{~V}$ for a $70 \mathrm{mV}(130 \mathrm{fC})$ threshold. For comparison, the working point of this RPC when equipped with ADULT cards was $10125 \mathrm{~V}$ for a threshold of $7 \mathrm{mV}$ (maxi-avalanche mode without FEE amplification). The HV difference is of 750 $\mathrm{V}$, in line with what was observed during preliminary tests with cosmic rays.

\subsection{Efficiency stability}

Figure 3 (left) shows the time dependence of the efficiency of the RPC equipped with FEERIC. Measurements demonstrate a very high efficiency $(\sim 97 \%)$ for both bending and non-bending planes. The stability is also evident for the three colliding systems characterizing the 2015 data taking. 

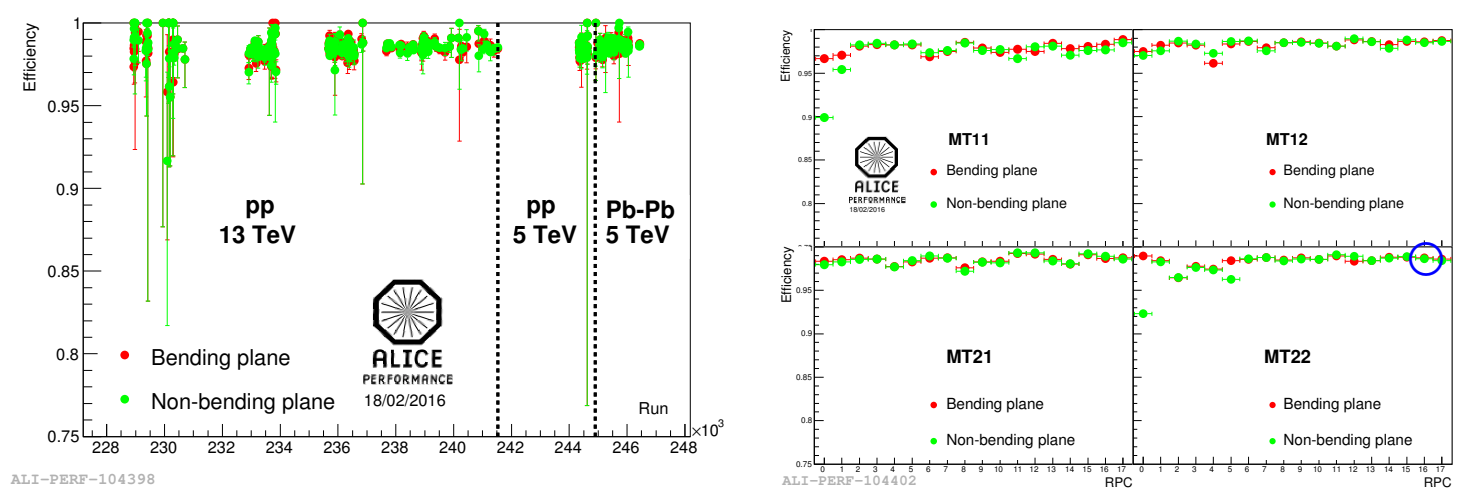

Figure 3. Left: efficiency of the RPC equipped with FEERIC measured in 2015. Right: average efficiency of the 72 RPCs composing the ALICE muon trigger. The blue circle indicates the chamber equipped with the new electronics. Red (green) points indicate the bending (non-bending) plane.

The comparison with the other chambers is shown in figure 3 (right). It displays the efficiency of the 71 RPCs still equipped with the non-amplified electronics and the chamber with the new front-end in the blue circle. The values are averaged over the whole 2015 . The efficiency of the chamber with FEERIC is perfectly in agreement with the results obtained for the other RPCs or slightly better.

\subsection{Dark current and dark rate time dependence}

Dark currents have been measured with dedicated runs in the absence of collisions, with the HV at their nominal values. The average dark current of the 71 RPCs equipped with ADULT at nominal voltage ranges from $2 \mu \mathrm{A}$ to $4 \mu \mathrm{A}$, increasing with time as can be seen in figure 4 (left). Conversely, the dark current of RPC with FEERIC is much lower $(\sim 0.6$ $\mu \mathrm{A})$ and stable throughout the data taking, as shown in the right part.
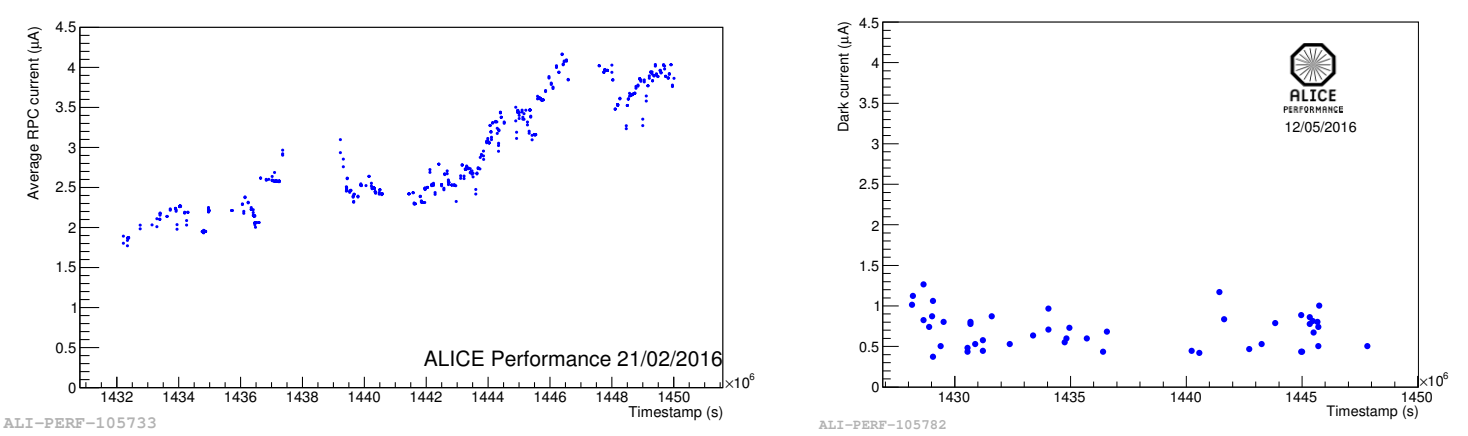

Figure 4. Average dark current drawn by the 71 RPCs equipped with ADULT (left) compared to the dark current of the RPC with FEERIC (right).

The dark rate has also been measured. This measurement is obtained from the strip scalers and hence it is dead time free. The average dark rate of RPCs with ADULT is below 
$0.05 \mathrm{~Hz} / \mathrm{cm}^{2}$ (figure 5, left) and stable in time, while the dark rate of RPC with FEERIC is larger, but usually below $0.1 \mathrm{~Hz} / \mathrm{cm}^{2}$.
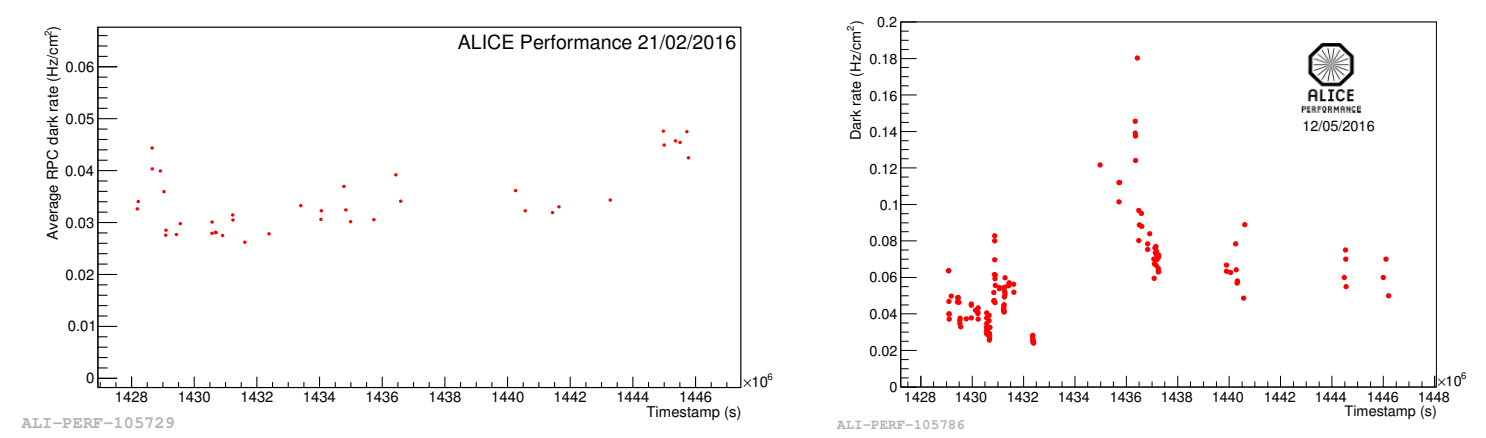

Figure 5. Average dark rate of the RPCs with ADULT (left) compared to the dark rate of the RPC with FEERIC (right).

\subsection{Evaluation of the charge reduction with FEERIC}

As previously mentioned, the main goal of this front-end electronics upgrade is to reduce the total charge produced in the gas gap, in order to prevent aging effects and to improve the rate capability. In order to evaluate the gain in charge obtained in the new working conditions, the RPC current has been measured at fixed interaction rate of $\sim 400 \mathrm{kHz}$ in July 2015 after subtraction of the dark component. Measurements have been performed at the $\mathrm{HV}$ value of $9375 \mathrm{~V}$, corresponding to the working point with FEERIC, and at the $\mathrm{HV}$ value of $10125 \mathrm{~V}$ (ADULT working point). The results are shown in figure 6, together with two intermediate HV measurements. The gain in current (and therefore in charge) obtained is more than a factor of 4 .

\section{Conclusions and perspectives}

In summary, a completely new front-end electronics has been designed with the aim to increase the rate capability and limit aging effects. The performance of one RPC equipped with the new cards have been studied in a dedicated cosmic data taking and in pp and $\mathrm{Pb}-\mathrm{Pb}$ collisions. It has been possible to lower the working point by $750 \mathrm{~V}$ maintaining an efficiency larger than $95 \%$. The main goal of the FEERIC upgrade project has been achieved: a gain in current (and therefore in total charge) of a factor 4 has been reached as compared to the present operations in maxi-avalanche. Performance related to cluster size and single counting rate are also within specifications. This study confirmed the robustness and the reliability on a long time scale of FEERIC in realistic conditions.

\section{References}

[1] ALICE Collaboration, The ALICE experiment at the CERN LHC, JINST 3 (2008) S08002.

[2] ALICE Collaboration, The forward muon spectrometer of ALICE: addendum to the technical proposal for a Large Ion Collider experiment at the CERN LHC, CERN-LHCC-96-032. 


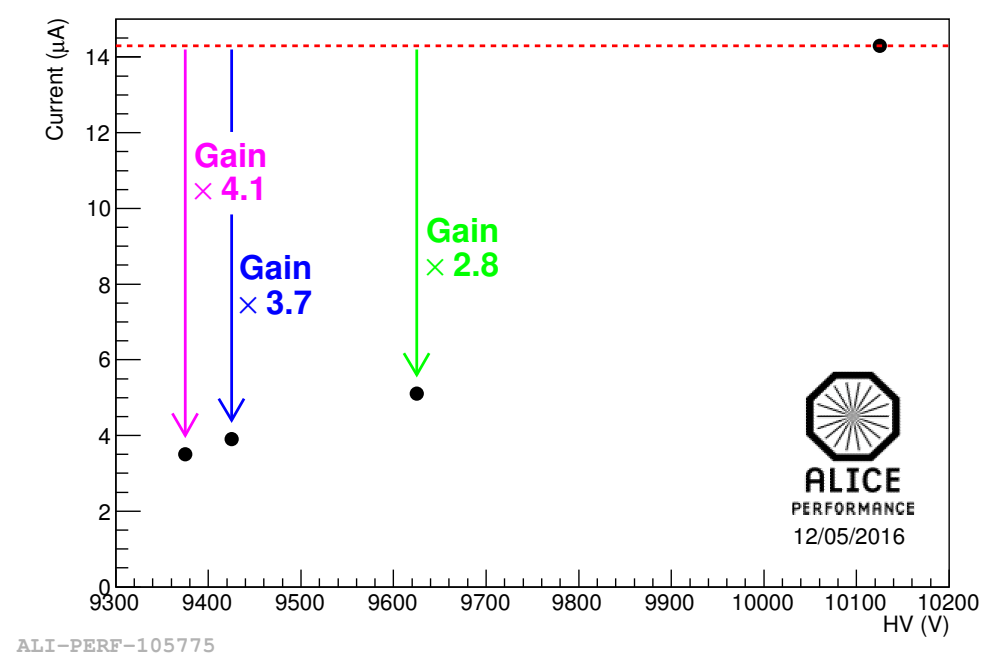

Figure 6. Current drawn in pp collisions by the RPC equipped with FEERIC at different HV. The gain with respect to the old working point is also shown.

[3] R. Arnaldi et al., Study of the resistive plate chambers for the ALICE Dimuon Arm, Nucl. Instr. and Meth. A 456 (2000) 73.

[4] R. Santonico et al., Development of resistive plate counters, Nucl. Instr. and Meth. A 187 (1981) 377.

[5] R. Arnaldi et al., A low-resistivity RPC for the ALICE dimuon arm, Nucl. Instr. and Meth. A $451(2000) 462$.

[6] R. Arnaldi et al., Beam and aging tests with a highly-saturated avalanche gas mixture for the ALICE p-p data taking, Nucl. Phys. B 158 (2006) 149.

[7] R. Arnaldi et al., Aging tests and chemical analysis of Resistive Plate Chambers for the trigger of the ALICE dimuon arm, Nucl. Instr. and Meth. A 533 (2004) 112.

[8] M. Gagliardi, Commissioning and first performance of the resistive plate chambers for the ALICE muon arm, Nucl. Instr. and Meth. A 661 (2012) S45.

[9] R. Arnaldi et al., Front-end electronics for the RPCs of the ALICE dimuon, IEEE Trans. Nucl. Sci. 52 (2005) 1176.

[10] F. Bossù et al., Performance of the RPC-based ALICE muon trigger system at the LHC, JINST 7 (2012) T12002.

[11] ATLAS Collaboration, The ATLAS Experiment at the CERN Large Hadron Collider, JINST 3 (2008) S08003.

[12] F. Giannini et al., An 8 channel GaAs IC front-end discriminator for RPC detectors, Nucl. Instr. and Meth. A 432 (1999) 440.

[13] CMS Collaboration, The CMS experiment at the CERN LHC, JINST 3 (2008) S08004.

[14] M. Abbrescia et al., New developments on front-end electronics for the CMS Resistive Plate Chambers, Nucl. Instr. and Meth. A 456 (2000) 143.

[15] P. Dupieux et al., Upgrade of the ALICE muon trigger electronics, JINST 9 (2014) C09013.

[16] R. Arnaldi et al., Final results of the tests on the resistive plate chambers for the ALICE muon arm, Nucl. Instr. and Meth. A 602 (2009) 740. 